Check for updates

Cite this: RSC Adv., 2017, 7, 41980

Received 16th June 2017

Accepted 31st July 2017

DOI: $10.1039 / \mathrm{c} 7 \mathrm{ra06709g}$

rsc.li/rsc-advances

\section{Microwave absorption performance of methylimidazolium ionic liquids: towards novel ultra-wideband metamaterial absorbers}

\author{
Jianhao Gong, (D) a Fulong Yang, ${ }^{a}$ Qunfeng Shao, ${ }^{a}$ Xiaodong He, ${ }^{a}$ Xiaoping Zhang, ${ }^{* a}$ \\ Shimin Liu, ${ }^{b}$ Liyun Tang ${ }^{c}$ and Youquan Deng (D)*b
}

\begin{abstract}
Here, for the first time, we propose a simple strategy to realize an ultra-wideband metamaterial absorber by using room temperature ionic liquids (ILS) which are a new class of organic salts containing purely anions and cations. At first, the microwave loss characteristics of methylimidazolium ILs were investigated. The results reveal that these ILs have large ionic conductivities and dielectric loss factors which means high conductive loss and dielectric loss of microwaves can both be caused, and the IL with a shorter carbon chain on cation has potential to produce a higher absorption. When the IL $[\mathrm{EMIm}]\left[\mathrm{N}(\mathrm{CN})_{2}\right]$ is properly arranged into periodic cylinders supported by a 3D-printed dielectric bracket, impedance matching can be enhanced in a wide band, and a strong absorption of microwave above $90 \%$ can be achieved within the whole frequency range of $8.4-29.0 \mathrm{GHz}$, which was verified in simulations and experiments. In addition, the IL-based metamaterial absorber can work efficiently at arbitrary polarization angles and wide incident angles from $0^{\circ}$ to $45^{\circ}$. Mechanism investigations show that the perfect absorption in ILs-based MMAs is mainly originated from the loss of dielectric polarization and induced ionic currents in ILs. Distinct from metamaterial absorbers based on water or other liquids, such IL-based metamaterial absorber can not only broaden the absorption bandwidth significantly, but also have potential application in a wider temperature range because of the exceedingly high thermal stabilities of ILs.
\end{abstract}

\section{Introduction}

Room temperature ionic liquids (ILs) are a class of organic salts that remain liquid at or near room temperature, comprised entirely of cations and anions. As a novel kind of green electrolyte $^{1,2}$ and soft material, ${ }^{3,4}$ ILs possess many excellent physical properties such as a broad liquid temperature range, high ionic conductivity, nonvolatile etc. ${ }^{5,6}$ Recently, researchers started to notice that ILs have great potential in microwave absorption due to their high density of dipoles. For example, Tang et al. ${ }^{7}$ measured the permittivities of four ILs in the solid state, i.e. $\mathrm{P}[\mathrm{VBBI}]\left[\mathrm{BF}_{4}\right], \quad \mathrm{P}[\mathrm{VBBI}][\mathrm{Sac}], \quad \mathrm{P}[\mathrm{VBBI}]\left[\mathrm{FeCl}_{4}\right]$, and $\mathrm{P}[\mathrm{VBTMA}]\left[\mathrm{BF}_{4}\right]$, and found that these ILs have higher dielectric loss factors $(0.18-0.37)$ than other commonly used polymers. ${ }^{8}$ Hence, they inferred that ILs could be a kind of candidate material for microwave absorption after designing their chemical structures. However, the absorption performance of other

${ }^{a}$ School of Information Science and Engineering, Lanzhou University, Tianshui South Road, Lanzhou 730000, China.E-mail: zxp@lzu.edu.cn

${ }^{b}$ Centre for Green Chemistry and Catalysis, Lanzhou Institute of Chemical Physics, Chinese Academy of Sciences, Tianshui Middle Road, Lanzhou, 730000, China. E-mail: ydeng@licp.cas.cn

'Institute of Applied Magnetism, Key Laboratory for Magnetism, Magnetic Materials of Ministry of Education, Lanzhou University, Lanzhou, 730000, China kinds of ILs have not been studied until now. And there is few study on the ILs-based microwave absorber as far as we know.

In the past decade, metamaterial absorbers (MMAs) are attracting more and more attention due to their extensive applications, such as photo-detectors, ${ }^{\mathbf{9 , 1 0}}$ solar cells, ${ }^{\mathbf{1 1 , 1 2}}$ thermal emitters, ${ }^{13,14}$ and image systems, ${ }^{15,16}$ etc. The first MMA was proposed by Landy et al. ${ }^{17}$ in 2008 , but only a single absorption peak at $11.8 \mathrm{GHz}$ with the absorptivity of $88 \%$ was obtained in their design. From then on, many efforts have been made to broaden the absorption bandwidth of MMAs. ${ }^{18-22}$ For example, Ding et $a .^{23}$ designed a wideband MMA with multi-layered metal-dielectric overlapping quadrangular frustum pyramids meta-atoms. Later, Long et al. ${ }^{\mathbf{2 4}}$ developed the multi-layered structure by deliberately regulating period length between adjacent unit cells, and more than $90 \%$ absorption in a wideband of $11 \mathrm{GHz}$ could be achieved. However, it is a complex and difficult process to stick multiple thin layers vertically. In addition, using magnetic materials such as MnTi substituted M-type barium ferrite as the substrates or resonators can also broaden the absorption band, ${ }^{25,26}$ but the fabrication of such magnetic materials is relatively complicated. In 2015, Yoo et $a .^{27}$ realized wideband absorption in MMA just by using a single-layered water droplets array, and more than $91 \%$ of average absorption ratio was obtained in very wide range of 10 $\mathrm{GHz}$ as the total thickness reached $4.5 \mathrm{~mm}$. Different from 
conventional solid-material-based MMAs, the liquid-based MMAs have advantage of easy processing, as well as the controllable absorption because of the tuneable shape and thickness of water. Meanwhile, water as a frequency dispersion material has free ions which is beneficial to enhance the absorption even weak. However, the narrow liquid temperature range of water $\left(0-100^{\circ} \mathrm{C}\right)$ would limit its application, especially at the relatively higher/lower temperatures. Therefore, it is desired to develop a novel liquid material for the metamaterial absorbers, and ILs are considered as a kind of ideal alternatives.

In this paper, a novel ILs-based metamaterial absorber (ILMMA) was realized for wideband absorption of microwave by taking advantage of the high electromagnetic loss property of methylimidazolium ILs. At first, conductivities and permittivities of seven methylimidazolium ILs with different anions and cations were measured and the results reveal that these ILs contain a number of free ions and anion-cation pairs and therefore have relative large conductivities and dielectric loss factors, which means the microwave absorption of ILs caused by conductive loss and dielectric polarization loss is high. And the dielectric properties of ILs is very sensitive to the ionic radii. Furthermore, the reflection loss of different ILs were calculated and the results reveal that the absorption performance of ILs are positively related to their microwave loss properties. Based on these characteristics of ILs, $[\mathrm{EMIm}]\left[\mathrm{N}(\mathrm{CN})_{2}\right]$ was selected due to its strong absorption of microwave. Then, by properly arranging $[\mathrm{EMIm}]\left[\mathrm{N}(\mathrm{CN})_{2}\right]$ into cylinder arrays supported by a 3D-printed dielectric bracket and suitably adjusting the cylinder sizes, effective impedance of the composite structure can be greatly tuned to match with the free space. So, microwave can propagate into the IL as much as possible and then dissipate. The simulative and experimental results show that such absorber has the capability of working in an ultra-wideband reaching to $20.6 \mathrm{GHz}$ with more than $90 \%$ absorptivity. Meanwhile, the absorber is valid to a wide range of incident and polarization angles. Additionally, the mechanism of wideband absorption was also investigated. It is demonstrated that low real part of permittivity and high absorption property of IL are critical factors for the strong wideband absorption. This is the first time that ILs are used in MMAs, which can not only overcome the limitations of water or its solutions, but also drastically extend the absorption bandwidth than water-dropletsbased MMA with the same thickness. ${ }^{27}$

\section{Experiments set up}

Seven methylimidazolium ILs with different anions and cations were selected for investigation, i.e. $[\mathrm{EMIm}][\mathrm{Ac}],[\mathrm{EMIm}]\left[\mathrm{BF}_{4}\right]$, $[\mathrm{EMIm}]\left[\mathrm{N}(\mathrm{CN})_{2}\right],[\mathrm{EMIm}]\left[\mathrm{NTf}_{2}\right],\left[\mathrm{BMIm}_{[}\right]\left[\mathrm{BF}_{4}\right],[\mathrm{HMIm}]\left[\mathrm{BF}_{4}\right]$ and [OMIm] $\left[\mathrm{BF}_{4}\right]$. These ILs were synthesized according to the literature method ${ }^{28,29}$ with purity $>99 \%$ and their structures are shown in Fig. 1. Before measurements, the ILs were kept in oil bath to obtain a constant temperature at $25{ }^{\circ} \mathrm{C}$. The conductivities $(\sigma)$ were measured by conductivity meter S230 SevenCompact ${ }^{\mathrm{TM}}$ with a precision of $+/-0.5 \%$. And the complex permittivities $\left(\varepsilon=\varepsilon^{\prime}+\varepsilon^{\prime \prime}\right)$ were measured by probe method on

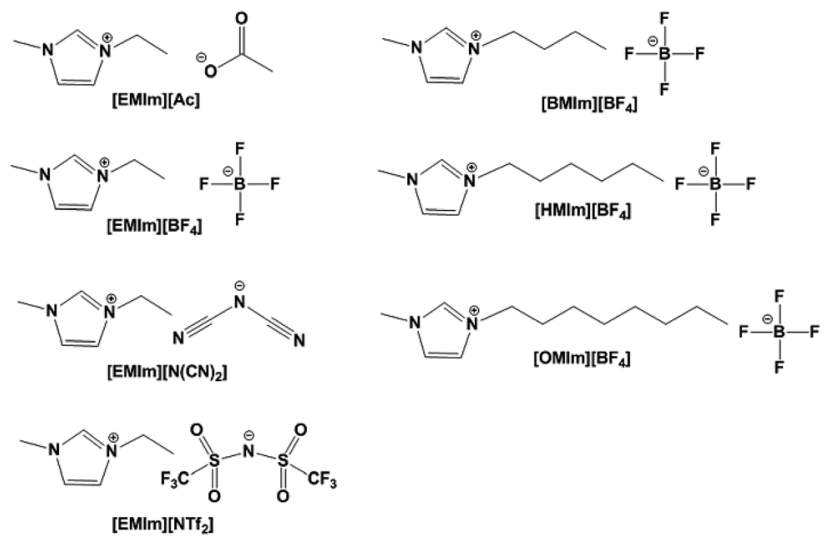

Fig. 1 Structures of methylimidazolium ILs with different anions and cations.

Agilent vector network analyser N5247A in the frequency range of $0.5-50 \mathrm{GHz}$.

The dielectric bracket for supporting IL cylinders contains an array of $12 \times 12$ units with a thickness of $1 \mathrm{~mm}$ which was fabricated by $3 \mathrm{D}$ printing technology. Here, we chose photopolymer DSM Somos 14120 for 3D printing and its dielectric constant is $3(1+0.02)$. Then, a copper film of $36 \mu \mathrm{m}$ was stuck at the bottom, and $285.1 \mu \mathrm{L}$ IL [EMIm] $\left[\mathrm{N}(\mathrm{CN})_{2}\right]$ was injected in every dielectric cylinder. Because the IL has a relative large viscosity as well as a bracket for supporting, the IL cylinders are relatively stable when the bracket was put flat on the textboard. The photograph of the ILMMA prototype is shown in Fig. 12(a). Free-space measurements were performed using standard horn antennas connected to the Agilent Network Analyzer N5247A in an electromagnetic wave anechoic chamber. The device was illuminated with a TE polarization beam from the transmitting horn, and another horn got the reflected microwave. The angle between two horns was set as $10^{\circ}$ to ensure a near normal incidence. For accuracy, the measurements were performed in three frequency bands of $2-8 \mathrm{GHz}, 8-18 \mathrm{GHz}, 18-30 \mathrm{GHz}$, respectively.

\section{Absorption performance of methylimidazolium ionic liquids}

In general, the absorption of microwave is mainly caused by dielectric loss, magnetic loss and conductive loss. ${ }^{30}$ For ILs, the main losses are generated via ionic currents ascribed to the finite conductivity and dielectric polarization due to the high density of anion-cation pairs. To select an IL in liquid state that have the capacity of strong absorption, the conductivities $(\sigma)$ and permittivities $(\varepsilon)$ of seven methylimidazolium ILs were measured firstly. As shown in Table 1, these ILs have conductivities ranging from 0.075 to $2.800 \mathrm{~S} \mathrm{~m}^{-1}$, which relies on the number and mobility of the charge carriers. It can be observed that IL has a decreased conductivity with increasing cabin chain length on cation, which could be explained by the enhanced ion cluster effect and the resulting increased viscosity of IL. $^{31}$ In addition, the effect of anions on conductivities mainly depends 
Table 1 Conductivities of different methylimidazolium ILS at $25^{\circ} \mathrm{C}\left(\mathrm{S} \mathrm{m}^{-1}\right)$

Cation [EMIm]

\begin{tabular}{llll}
\hline$[\mathrm{Ac}]$ & {$\left[\mathrm{NTf}_{2}\right]$} & {$\left[\mathrm{BF}_{4}\right]$} & {$\left[\mathrm{N}(\mathrm{CN})_{2}\right]$} \\
\hline 0.281 & 0.920 & 1.405 & 2.800 \\
\hline Anion $\left[\mathrm{BF}_{4}\right]$ & & & \\
\hline$[\mathrm{EMIm}]$ & {$[\mathrm{BMIm}]$} & {$[\mathrm{HMIm}]$} & {$[\mathrm{OMIm}]$} \\
\hline 1.405 & 0.409 & 0.151 & 0.075
\end{tabular}

on the strength of interaction between anions and cations. ${ }^{32}$ Among these examined ILs, [EMIm] $\left[\mathrm{N}(\mathrm{CN})_{2}\right]$ possesses the largest conductivity of $2.800 \mathrm{~S} \mathrm{~m}^{-1}$. Compared with tap-water $\left(\sigma=0.049 \mathrm{~S} \mathrm{~m}^{-1}\right),[\mathrm{EMIm}]\left[\mathrm{N}(\mathrm{CN})_{2}\right]$ has a higher conductivity and therefore can produce larger ionic currents, which could induce more mass transport to enhance the absorption of microwave greatly. ${ }^{27}$

The dielectric loss properties of ILs are characterized with the complex permittivities. The complex permittivities of seven methylimidazolium ILs with different anions and cations were

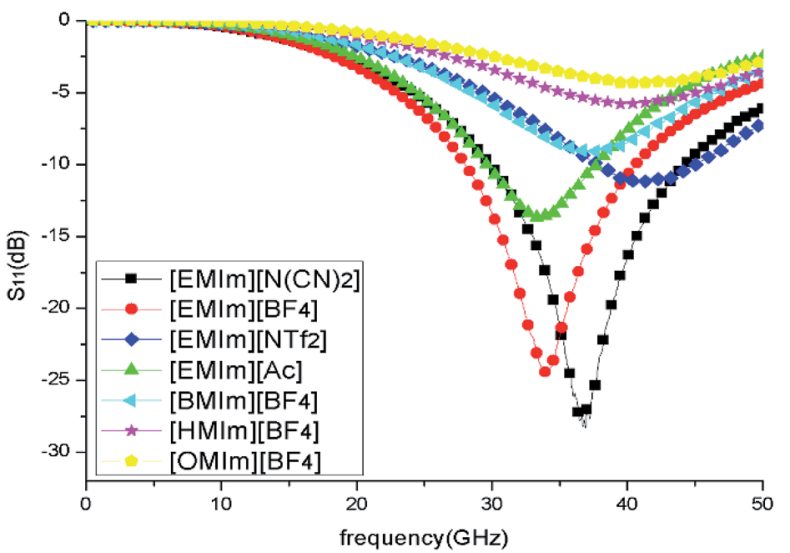

Fig. 3 Reflection loss of different methylimidazolium ILs layers with thickness of $1 \mathrm{~mm}$ on copper surface.

measured and the effect of ILs structures on their permittivities was investigated. Fig. 2(a) and (b) show the real part of permittivities $\left(\varepsilon^{\prime}\right)$ and loss tangent $\left(\tan \delta=\varepsilon^{\prime \prime} / \varepsilon^{\prime}\right)$ of ILs with different anions. It can be observed that the values of $\varepsilon^{\prime}$ and $\tan \delta$ decline with increasing frequency, which may result from the lag between dipole rotation in ILs and the oscillation of the
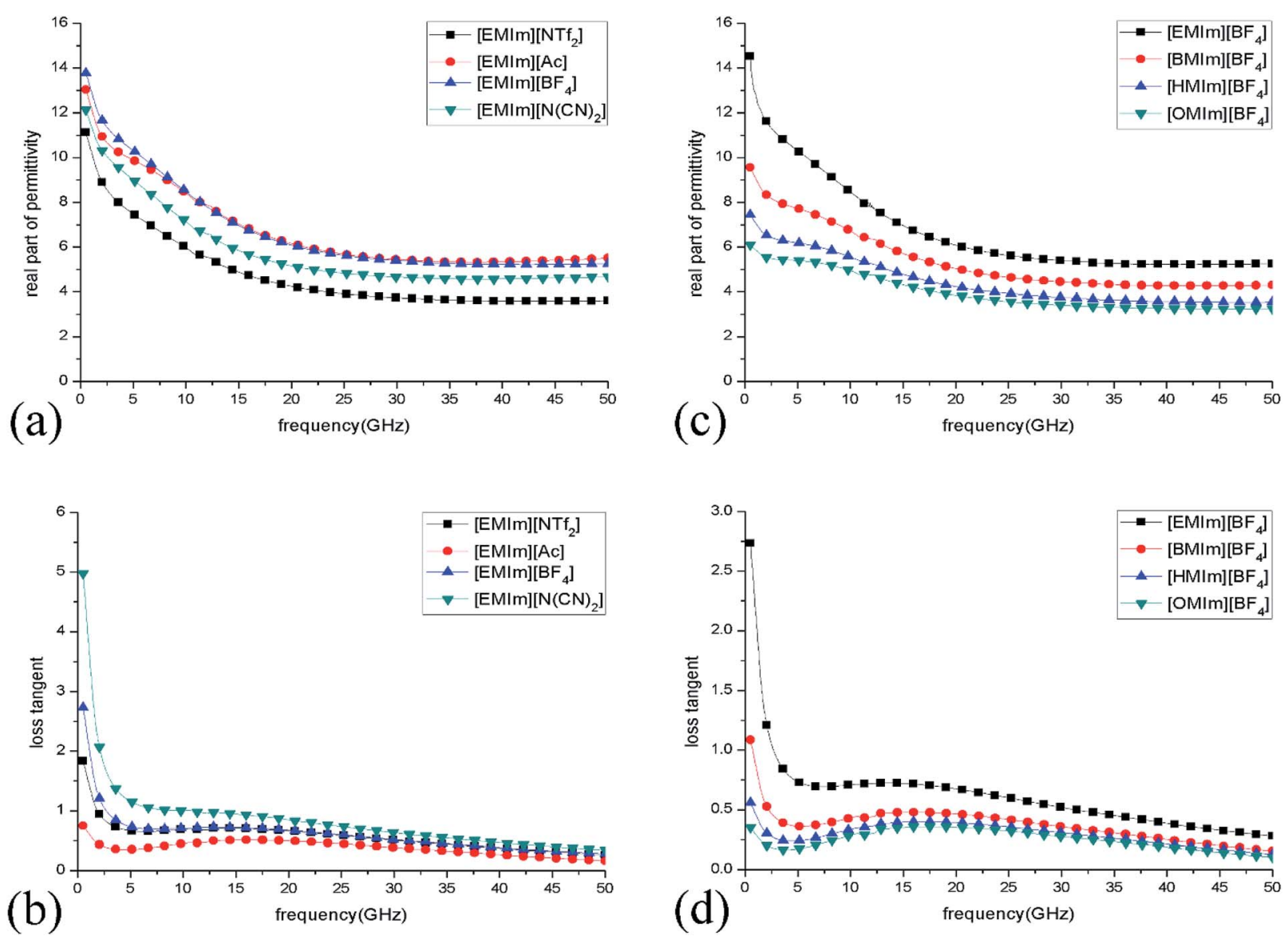

Fig. 2 (a) Real part of permittivity and (b) loss tangent of methylimidazolium ILs with different anions. (c) Real part of permittivity and (d) loss tangent of methylimidazolium ILs with different cations. 
electromagnetic field at high frequency. ${ }^{33}$ This remarkable dispersion property of ILs is beneficial to wideband absorption. ${ }^{34}$ On the one hand, the obtained results show that these ILs have relatively low $\varepsilon^{\prime}$ from 3.19 to 14.52 (about $1 / 6$ lower than water or water-based solutions), which means the dielectric resonance effect in ILs is weak. With increase of the anion radius, $\varepsilon^{\prime}$ of different ILs decrease apparently, i.e. [EMIm] [Ac] > $[\mathrm{EMIm}]\left[\mathrm{BF}_{4}\right]>[\mathrm{EMIm}]\left[\mathrm{N}(\mathrm{CN})_{2}\right]>[\mathrm{EMIm}]\left[\mathrm{NTf}_{2}\right]$. On the other hand, the $\tan \delta$ are quite large (0.16-5.91) which indicates that high dielectric loss can be caused in these ILs.

Also, it is necessary to examine the changes of permittivities of ILs with different cations. As shown in Fig. 2(c) and (d), with increase of carbon chain length on cation, the $\varepsilon^{\prime}$ and $\tan \delta$ of different ILs decrease obviously: $[\mathrm{EMIm}]\left[\mathrm{BF}_{4}\right]>[\mathrm{BMIm}]\left[\mathrm{BF}_{4}\right]>$ $[\mathrm{HMIm}]\left[\mathrm{BF}_{4}\right]>[\mathrm{OMIm}]\left[\mathrm{BF}_{4}\right]$, because IL with a larger cation radius possesses the larger dipole moment, which makes polarization more difficult in high frequency electromagnetic field. Based on above results, we can conclude that as ionic radii increase, $\varepsilon^{\prime}$ decrease. With increase of the cation radii, conductivities decrease and the values of $\tan \delta$ are positively related to the conductivities. For the electric loss dielectric, relatively high conductivity and large $\tan \delta$ are favourable to absorbing microwave. Among the studied ILs, [EMIm] $\left[\mathrm{N}(\mathrm{CN})_{2}\right]$ possesses the highest conductivity of $2.8 \mathrm{~S} \mathrm{~m}^{-1}$ as well as the largest loss tangent declines from 5.91 to 0.34 in the frequency range of $0.5-50 \mathrm{GHz}$ which indicates that it could produce the biggest conductive loss and dielectric loss simultaneously.

To clearly reveal the absorption performance of different ILs, the reflection loss of seven methylimidazolium ILs with different anions and cations were investigated in the frequency range of $0.5-50 \mathrm{GHz}$. The ILs were covered on the copper surface with a thickness of $1 \mathrm{~mm}$. Under normal incident of the plane waves, the reflection loss $\left(S_{11}\right)$ of ILs layers were calculated from their relative permeabilities and permittivities based on the transmission line theory. ${ }^{35}$ As shown in Fig. 3, the IL [EMIm] $\left[\mathrm{N}(\mathrm{CN})_{2}\right]$ has the lowest reflection which means its absorption is the strongest. On the contrary, the absorption of IL $[\mathrm{OMIm}]\left[\mathrm{BF}_{4}\right]$ is the weakest due to its low dielectric loss and conductive loss. The calculated results also reveal that the absorption performance of these ILs are positively related to their microwave loss

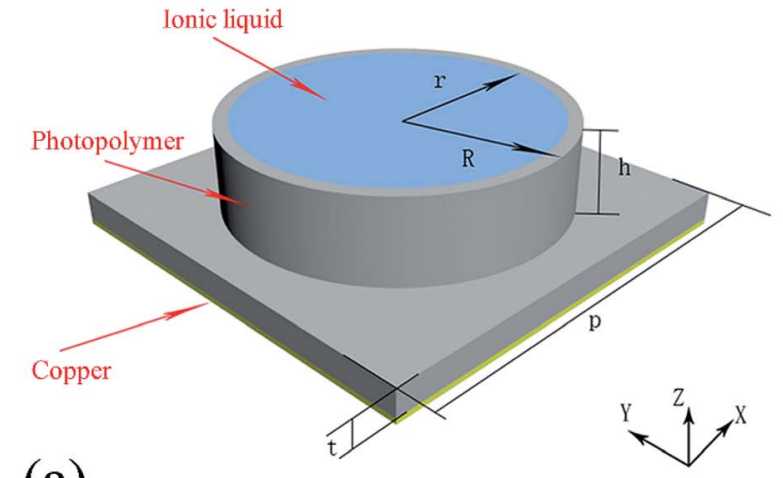

(a)

Fig. 4 (a) Schematic of an ILMMA unit cell and (b) the periodic array. properties. So, the IL $[\mathrm{EMIm}]\left[\mathrm{N}(\mathrm{CN})_{2}\right]$ was chosen to construct the metamaterial absorber due to its best absorption performance.

\section{Design of ultra-wideband ILMMA}

\section{Design of the periodic structure}

In order to realize impedance matching preferably, the IL $[\mathrm{EMIm}]\left[\mathrm{N}(\mathrm{CN})_{2}\right]$ was arranged into periodic cylinders instead of a homogeneous coating layer on metal surface. On the one hand, cylindrical shape is propitious to achieve polarization insensitivity due to the rotational symmetry. On the other hand, the IL was designed as periodic array because the average $\varepsilon^{\prime}$ of composite structure will decrease as the IL combined with low dielectric constant substrates like air. Fig. 4(a) shows the schematic of a unit cell of the proposed metamaterial absorber based on IL. The unit period was much smaller than the longest wavelength studied here $(50 \mathrm{~mm})$. And the IL cylinders were supported by a metal-backed dielectric bracket with a thickness of $1 \mathrm{~mm}$ to maintain their shapes. Additionally, the bottom copper layer was used for blocking the propagation of electromagnetic waves which has a thickness of $36 \mu \mathrm{m}$ and conductivity of $5.8 \times 10^{7} \mathrm{~S} \mathrm{~m}^{-1}$.

The commercial simulation software CST Microwave Studio 2014® was applied to calculate the absorption spectra in frequency range of $6-30 \mathrm{GHz}$. IL $[\mathrm{EMIm}]\left[\mathrm{N}(\mathrm{CN})_{2}\right]$ with a conductivity of $2.8 \mathrm{~S} \mathrm{~m}^{-1}$ and frequency dispersive permittivity was employed in this simulation. The unit cell was set as periodic boundary conditions on the $x-y$ plane and the microwave propagated along the $-z$ direction. The polarization of the electric field vector was along the $y$-axis. The finite element analysis was adopted to optimize parameters of the unit cell. In order to design an efficient microwave absorber, the reflection and transmission of the incident wave must be minimized. In this structure, there is no transmission because of the presence of a metal ground plane. So, the absorption can be defined as $A=1-R$ here, where $A$ and $R$ represent absorption and reflection respectively. $R$ can be calculated with the $S$-parameter, namely $\left|S_{11}\right|^{2}$. Therefore, $A$ can be obtained from the equation: $A=1-\left|S_{11}\right|^{2}$.

\section{(b)}

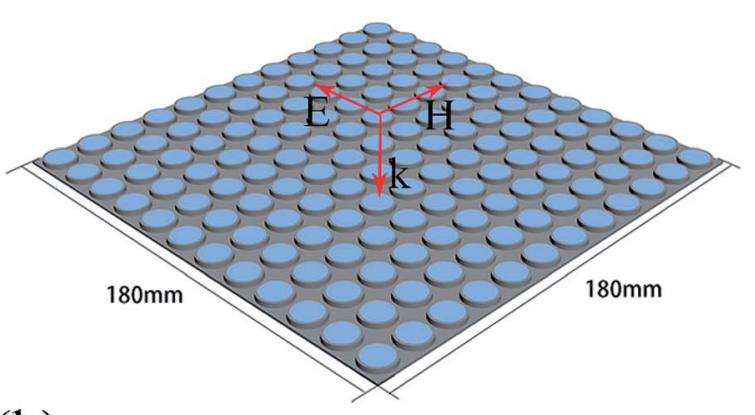



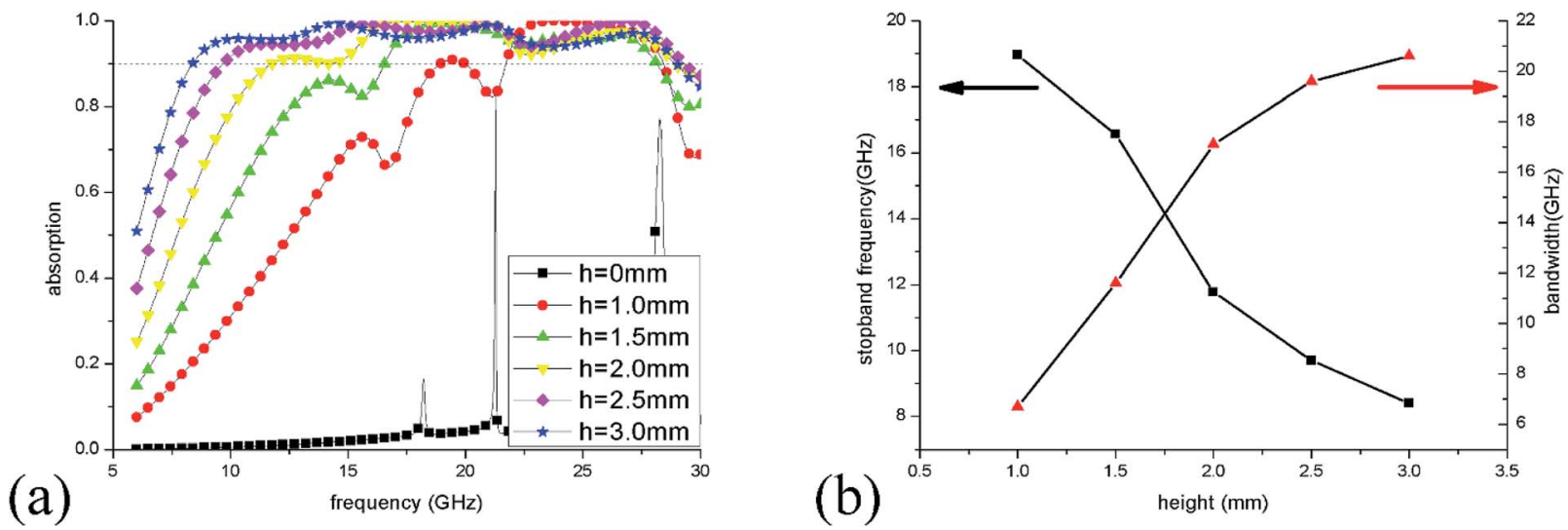

Fig. 5 Changes of microwave absorption by height of IL cylinders. (a) Changes of absorption spectra when changing height of IL cylinders by 6 steps $(0,1.0,1.5,2.0,2.5$ and $3.0 \mathrm{~mm}$ ) and fixing radius as $5.5 \mathrm{~mm}$. (b) Changes of stopband frequency and absorption bandwidth with cylinder height increases.

\section{Simulation results and numerical analysis}

To optimize the unit sizes of IL cylinders, a three-step strategy was adopted to obtain a broadband perfect absorption in the frequency range of 6-30 GHz. At first, the height of the IL cylinder was changed from 0 to $3 \mathrm{~mm}$ when its radius was kept at $5.5 \mathrm{~mm}$. The simulation results of absorption spectra are given in Fig. 5(a). When there is no IL injected into the dielectric

(a)
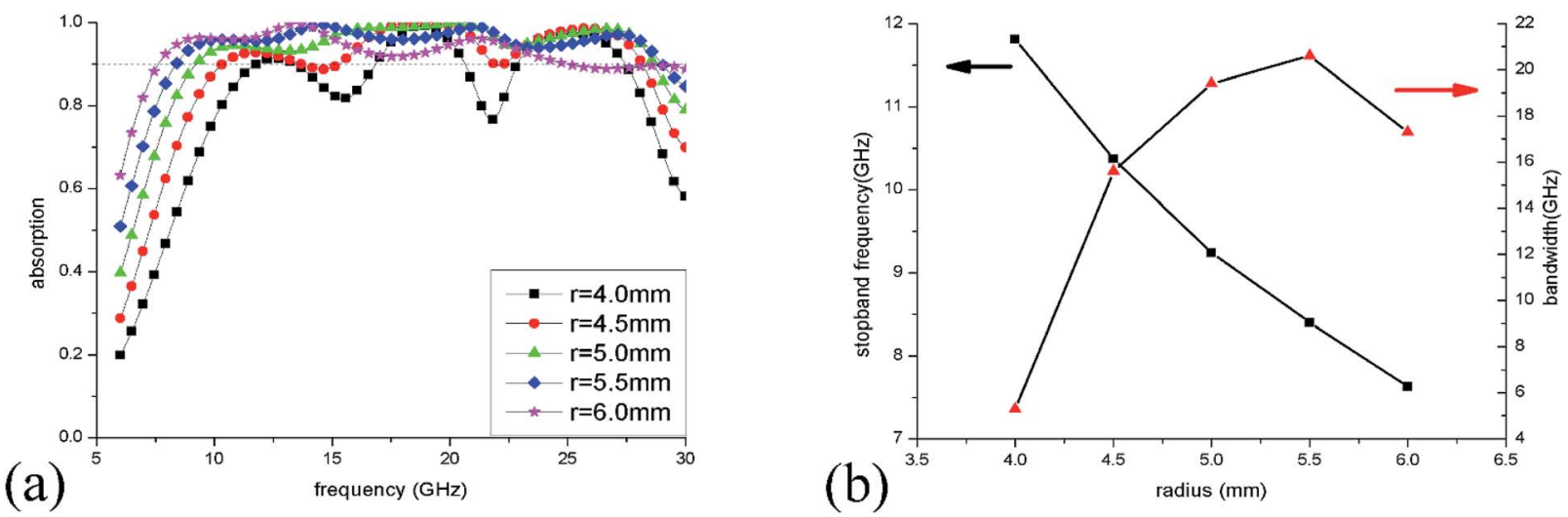

Fig. 6 Changes of microwave absorption by diameter of IL cylinders. (a) Changes of absorption spectra when changing radius of IL cylinders by 5 steps $(4.0,4.5,5.0,5.5$ and $6.0 \mathrm{~mm}$ ) and fixing height as $3 \mathrm{~mm}$. (b) Changes of stopband frequency and absorption bandwidth with cylinder radius increases.
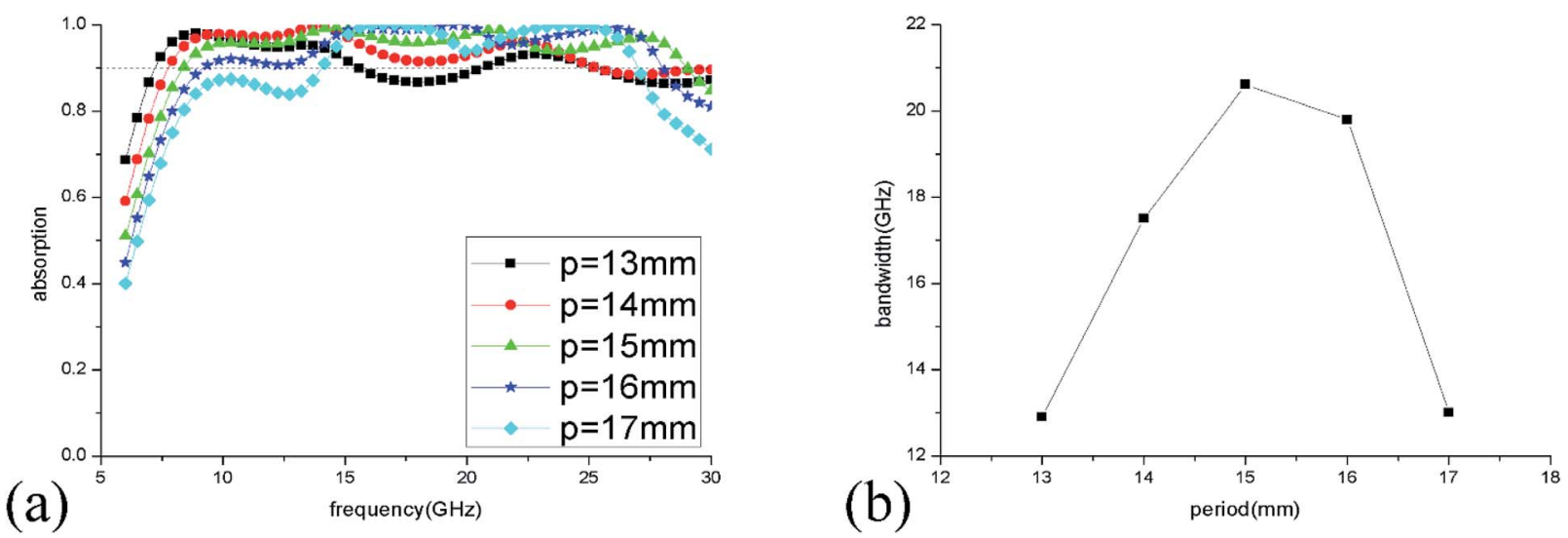

Fig. 7 Changes of microwave absorption by unit period. (a) Absorption spectra of ILMMA at different unit periods $(13,14,15,16$, and $17 \mathrm{~mm})$ when cylinder height is $3 \mathrm{~mm}$ and radius is $5.5 \mathrm{~mm}$. (b) Changes of the absorption bandwidth with the period increases. 

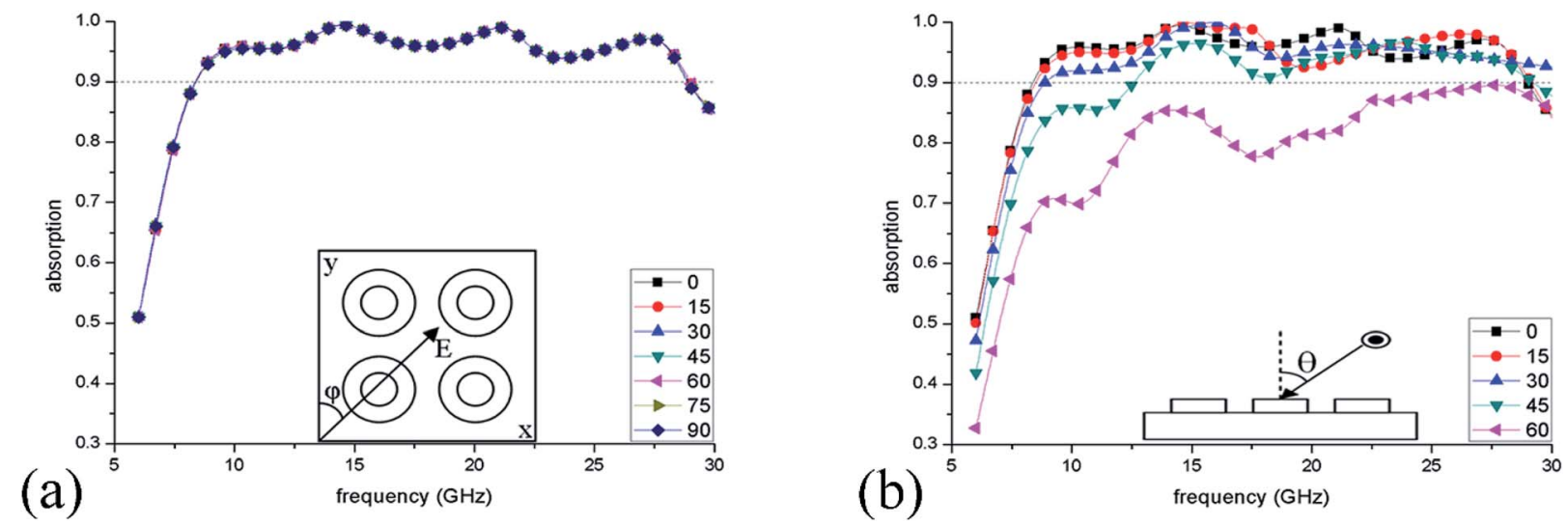

Fig. 8 Absorption spectra of the ILMMA at different (a) polarization angles $(\varphi)$ of $0-90^{\circ}$ and (b) incident angles $(\theta)$ of $0-60^{\circ}$

bracket, only two narrow absorption perks at $21.3 \mathrm{GHz}$ and 28.3 $\mathrm{GHz}$ are obtained, which are ascribed to dielectric absorption of the substrate. While, as the height of IL is more than $1 \mathrm{~mm},>90 \%$ absorption in a wide frequency range could be observed, suggesting that IL plays a key role in the microwave absorption. As the IL height increase, the absorption bandwidth becomes wider and wider. At the same time, the stopband frequency (the first frequency with absorptivity more than $90 \%$ ) shifts from $18.9 \mathrm{GHz}$ to $8.4 \mathrm{GHz}$ as the height adds from 0 to $3 \mathrm{~mm}$ [Fig. 5(b)], which results from the longer path of longitudinal currents induced in IL and the corresponding lower absorption frequency.

The radius of the IL cylinder is also an important aspect affecting the absorption performance. Fig. 6(a) shows the absorption spectra of ILMMA with various radii from $4 \mathrm{~mm}$ to $6 \mathrm{~mm}$ by fixing the height as $3 \mathrm{~mm}$. When the radius is $4.0 \mathrm{~mm}$, there are only three wide absorption peaks and the bandwidth with absorptivity more than $90 \%$ is discrete. For radius is more than $5 \mathrm{~mm}$, the absorber shows a wider and consecutive absorption band. However, as the radius reaches to $6 \mathrm{~mm}$, the absorption bandwidth decreases remarkably, which can be explained by the impedance mismatch. Fig. 6(b) plots the changes of stopband frequency and bandwidth with increase of radius. When the radius is set as $4.0 \mathrm{~mm}, 4.5 \mathrm{~mm}, 5.0 \mathrm{~mm}$, $5.5 \mathrm{~mm}$ and $6.0 \mathrm{~mm}$, the stopband frequency appears at $11.8 \mathrm{GHz}, 10.4 \mathrm{GHz}, 9.2 \mathrm{GHz}, 8.4 \mathrm{GHz}$ and $7.6 \mathrm{GHz}$, respectively. It is obvious that the smaller cylinder radius corresponds to lower stopband frequency.

The absorption spectra of ILMMA were also calculated as the unit period changed from 13 to $17 \mathrm{~mm}$. As shown in Fig. 7(a), with increase of the period, the absorption at low frequency decrease gradually. For the period is less than $15 \mathrm{~mm}$, the absorption at high frequency increase with increase of the period. While the period is more than $15 \mathrm{~mm}$, the absorption at high frequency decrease as the period increases. So, as shown in Fig. 7 (b), the $>90 \%$ absorption bandwidth first ascend and then descend with the adding of unit period. When the period is $15 \mathrm{~mm}$, the ILMMA has the widest absorption bandwidth. Comprehensively, as the period of IL cylinder is set as $15 \mathrm{~mm}$ with $3 \mathrm{~mm}$ height and $5.5 \mathrm{~mm}$ radius, the widest absorption band of $20.6 \mathrm{GHz}$ could be achieved. So, the optimum parameters were used in the following investigation.

Next, the dependence of absorption on the polarization and incident angle were investigated. Fig. 8(a) shows the simulated absorption spectra when the polarization angle $(\varphi)$ is varied from $0^{\circ}$ to $90^{\circ}$, and the results reveal that such IL-based absorber is polarization insensitive due to the rotational symmetry. Fig. 8(b) plots the absorption spectra within varied incident angles $(\theta)$ from $0^{\circ}$ to $60^{\circ}$. It can be found that even if the incident angle is $45^{\circ}$, the ILMMA still displays a wide $>90 \%$ absorption band of $12.57-29.21 \mathrm{GHz}$, indicating that this structure is conducive to realize incident angle insensitivity. (a)

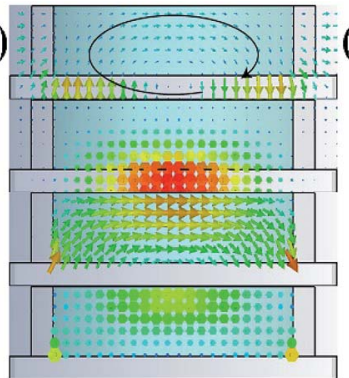

(b)
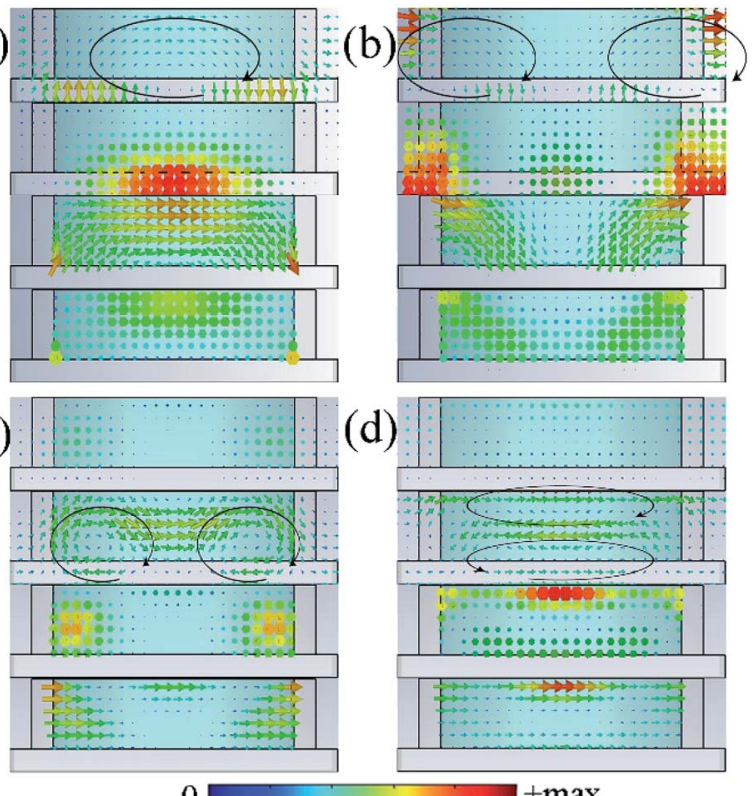

(d)

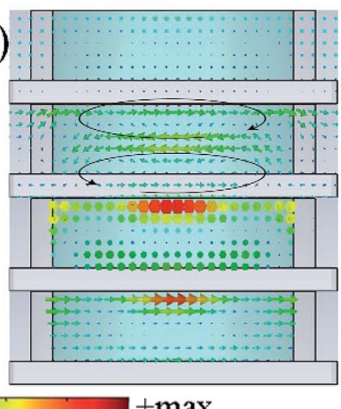

(c)

Fig. 9 Vector distributions of the electric field, magnetic field, induced currents and power loss density at resonance frequencies: (a) 10.0 GHz, (b) $14.5 \mathrm{GHz}$, (c) $21.1 \mathrm{GHz}$ and (d) $27.2 \mathrm{GHz}$. (a) and (b) are $y-z$ cutting planes along with the electric field orientation, (c) and (d) are $x-z$ cutting planes along with the magnetic field orientation. 

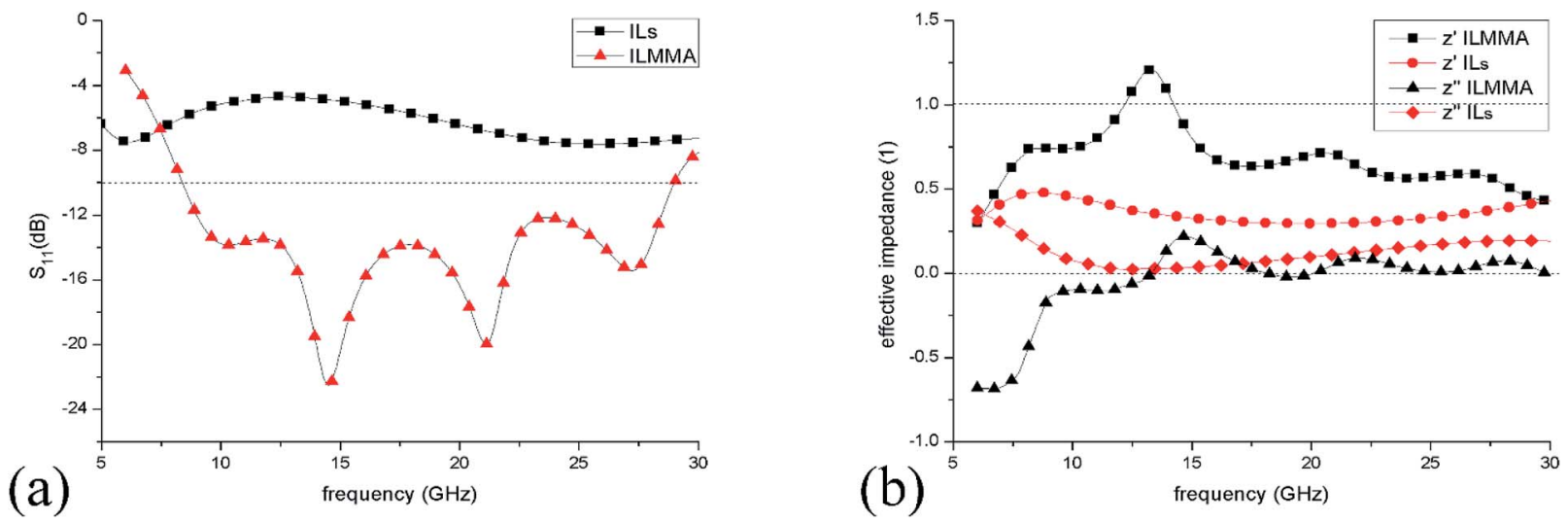

Fig. 10 (a) Reflection spectra of IL layer of $4 \mathrm{~mm}$ on copper surface and the corresponding ILMMA in frequency range of 6-30 GHz. (b) Calculated effective impedances of IL layer and ILMMA.

According to the results of absorption spectra in Fig. 5(a), 4 wide absorption perks around $10.0 \mathrm{GHz}, 14.5 \mathrm{GHz}, 21.1 \mathrm{GHz}$ and $27.2 \mathrm{GHz}$ could be observed, which indicates resonances occur in these frequencies. To deeply understand the physical mechanism of the wideband absorption, vector distributions of the electric field, magnetic field, current density and power loss density at resonance frequencies were investigated. As shown in Fig. 9(a) and (b), the loop-shaped electric field lines can be found at the first two peaks $(10.0 \mathrm{GHz}$ and $14.5 \mathrm{GHz})$ which are equivalent to magnetic dipoles in the far field, leading to the magnetic resonances. ${ }^{36}$ For the first peak at $10.0 \mathrm{GHz}$, magnetic field is mainly concentrated in the dielectric layer beneath the IL cylinders. While for the second peak at $14.5 \mathrm{GHz}$, the main magnetic and electric field is observed between adjacent units. In contrast, the magnetic field loops appear at $21.1 \mathrm{GHz}$ and $27.2 \mathrm{GHz}$ corresponding to electric resonances [Fig. 9(c) and (d)]. For absorption peak at $21.1 \mathrm{GHz}$, the magnetic field loops and electric field are localized in the left and right sides of the unit, while at $27.2 \mathrm{GHz}$ they are mainly confined in the upper and lower layers of IL. These behaviours show that different resonant modes are excited at the absorption peak frequencies even though weak. These resonances in the structure cause a dramatic change in effective impedance, resulting in a better impedance matching. At the same time, microwave is greatly concentrated and oscillating in the composite structures due to the resonances and ionic currents are induced in the IL. As shown in Fig. 9, the induced currents have same oscillation orientation and concentration area with the excitation electric field which is mainly influenced by the relative high conductivity of the IL. For the IL [EMIm] $\left[\mathrm{N}(\mathrm{CN})_{2}\right]$, it contains not only anion-cation pairs forming electric dipoles but also appreciable amounts of free ions. Under the radiation of external electromagnetic field, dipoles in IL are polarized and oscillate with the electric field. Simultaneously, the movement of free ions forms ionic currents. Finally, the electromagnetic energy is converted to heat by the dielectric loss and current loss in IL and subsequently dissipated. This can be verified by the same distribution area of the induced currents and the power loss density in Fig. 9.

In addition, reflection loss and normalized impedance of the IL ([EMIm] $\left.\left[\mathrm{N}(\mathrm{CN})_{2}\right]\right)$ layer on copper and the designed ILMMA were calculated for comparison. For IL layer on metal surface, the impedance was calculated from the relative permeability and permittivity, ${ }^{35}$ while for ILMMA, its effective impedance was retrieved from $S_{11} \cdot{ }^{37}$ Fig. 10(a) shows that as IL covers whole
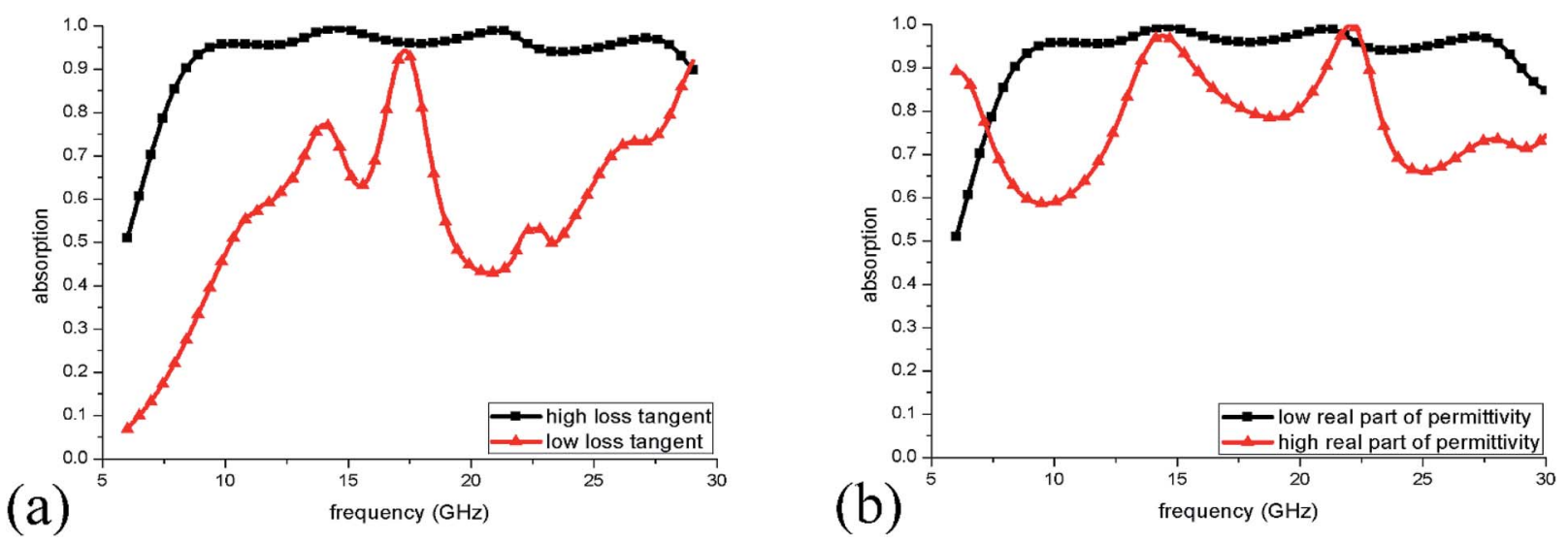

Fig. 11 (a) Absorption spectra of ILMMA with decreased loss tangent of IL. (b) Absorption spectra of ILMMA with increased real part of permittivity of IL. 

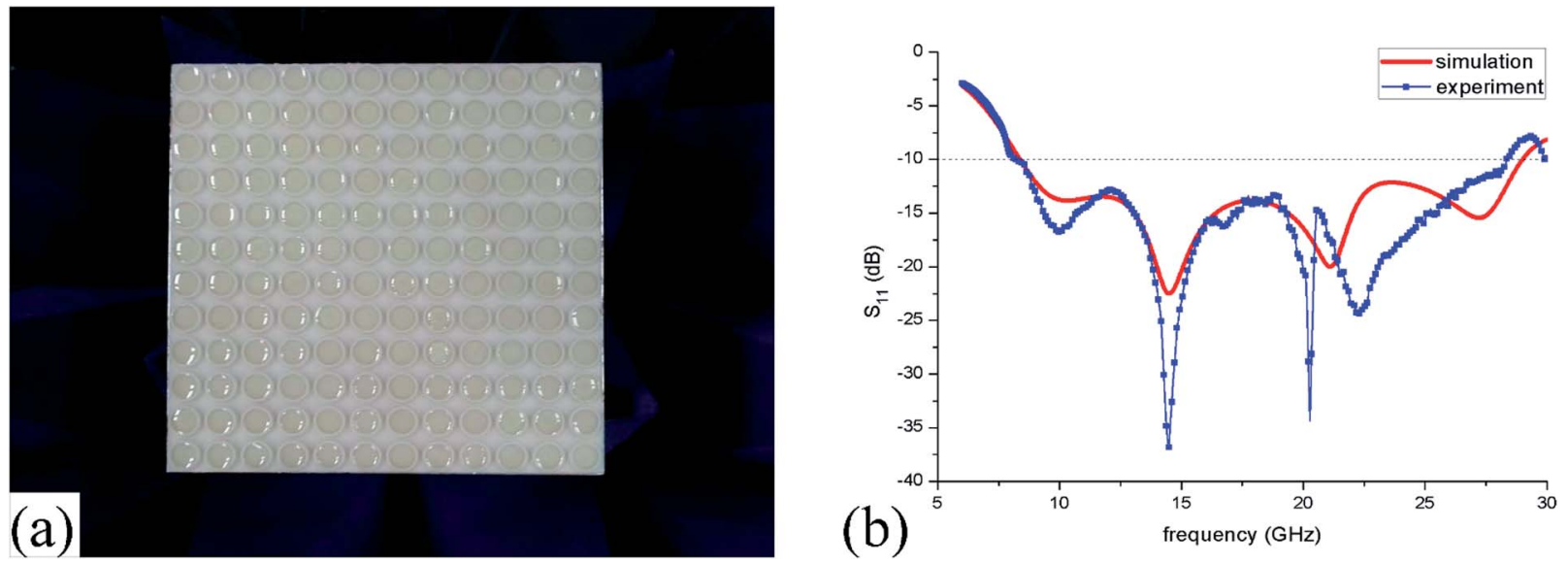

Fig. 12 (a) Photograph of the ILMMA prototype (b) simulated and measured reflection coefficient $\left(S_{11}\right)$.

surface of metal, the reflection is more than $-8 \mathrm{~dB}$ in frequency range of $5-30 \mathrm{GHz}$ which indicates a relative weak absorption. In contrast, periodical IL cylinders show a low reflection below $-10 \mathrm{~dB}$. The effective impedances of the ILMMA and IL layer under normal incidence are given in Fig. 10(b). It can be found that the real part of impedance $\left(z^{\prime}\right)$ of ILMMA is much larger than IL layer and closer to 1, meanwhile, the imaginary part is smaller and closer to 0 . This result proves that arrangement of ionic liquids into cylinders can effectively improve the impedance matching.

Finally, to explain the causes of wideband absorption more clearly, we present the absorption spectra when the loss tangent of IL decrease to one-tenth of what they had been. In this case, the dielectric loss of IL was very weak and the absorption was mainly caused by the induced ionic currents in IL. As shown in Fig. 11(a), when the loss tangent is low, the total absorption of ILMMA becomes weak in the wide frequency range. This behaviour indicates that the dielectric loss of IL plays an important role in perfect absorption. For example, at $10.2 \mathrm{GHz}$, the dielectric loss and current loss each account for half of the absorption. While at $17.3 \mathrm{GHz}$, the absorption mostly caused by dielectric loss. Fig. 11(b) shows changes of absorption spectra as the $\varepsilon^{\prime}$ of IL was set as ten times larger by keeping the tangent loss unchanged. It can be observed that the perfect absorption bandwidth becomes much narrow, which results from the excitation of strong dielectric resonances in the high permittivity material..$^{38}$ The above results reveal that ILs are a kind of ideal liquid materials for constructing wideband metamaterial absorbers because of the low $\varepsilon^{\prime}$ and high density of ions and dipoles.

\section{Experiment results}

The measured reflection spectra are shown in Fig. 12(b) and the simulated one is also plotted here for comparison. It can be found that the measured absorption bandwidth is $8.2-28.3 \mathrm{GHz}$ which only has a small relative error of $2.4 \%$ compared with the simulated results. Meanwhile, they both have four absorption peaks in the wide frequency range of 6-30 GHz. Before $18 \mathrm{GHz}$, the simulated and measured lines have peaks at the same frequency of $10.0 \mathrm{GHz}$ and $14.5 \mathrm{GHz}$. Remarkably, the measured line has a lower reflection than the simulated result which is primary due to the little changed volume of IL and environment absorption. However, measured peaks at more than $18 \mathrm{GHz}$ are different from the simulated results which may result from two main reasons. One is that the horn size dose not completely match with the radiated wavelength which could bring some deviations at the high frequency (>18 GHz). While another reason is the more sensitive conditions of the mismachining tolerance at high frequencies.

\section{Conclusions}

In conclusion, we investigated the absorption performance of methylimidazolium ionic liquids and realized an ultrawideband metamaterial absorber based on ILs for the first time. The measured results show that methylimidazolium ILs have high electromagnetic loss properties which are mainly influenced by ionic radii, indicating a great potential application in microwave absorption. By arranging IL [EMIm] $\left[\mathrm{N}(\mathrm{CN})_{2}\right]$ into periodic cylinders, impedance matching can be improved and $>90 \%$ absorption was obtained in the wide frequency range from 8.4 to $29.0 \mathrm{GHz}$. Such IL-based metamaterial absorber is insensitive to polarization and can achieve wide incident angle absorption. Moreover, the main causes of the wideband absorption have been investigated. Adopting ILs in metamaterials provides a simple route to achieve wideband absorption of microwave, and ILs as a novel class of liquid materials are promising to be applied in microwave absorption, especially in the flexible materials. To fabricate the structure easily, we adopted $3 \mathrm{D}$ printing technology and the bracket is made of photopolymer which is difficult to bend. In the future research, a flexible absorber based on ionic liquids could be realized when the bracket is constructed by a flexible material.

\section{Conflicts of interest}

There are no conflicts of interest to declare. 


\section{Acknowledgements}

This work was supported by the National Key Research and Development Program of China (2017YFA0403101) and the Fundamental Research Funds for the Central Universities (lzujbky-2015-315, lzujbky-2016-141).

\section{Notes and references}

1 M. Galiński, A. Lewandowski and I. Stepniak, Electrochim. Acta, 2006, 51, 5567-5580.

2 A. Balduccia, W. A. Hendersonc, M. Mastragostinoa, S. Passerinic, P. Simonb and F. Soavia, Electrochim. Acta, 2005, 50, 2233-2237.

3 T. Fukushima and T. Aida, Chem.-Eur. J., 2007, 13, 50485058.

4 S. Tang, A. Babai and A.-V. Mudring, Angew. Chem., 2008, 47, 7631-7634.

5 R. D. Rogers and K. R. Seddon, Science, 2003, 302, 792-793. 6 M. Armand, F. Endres, D. R. MacFarlane, H. Ohno and B. Scrosati, Nat. Mater., 2009, 8, 621-629.

7 J. Tang, M. Radosz and Y. Shen, Macromolecules, 2008, 41, 493-496.

8 B. Riddle, J. Baker-Jarvis and J. Krupka, IEEE Trans. Microwave Theory Tech., 2003, 51, 727-733.

9 F. Xia, T. Mueller, Y.-m. Lin, A. Valdes-Garcia and P. Avouris, Nat. Nanotechnol., 2009, 4, 839-843.

$10 \mathrm{~J}$. Le Percheca, Y. Desieres, N. Rochat and R. Espiau de Lamaestre, Appl. Phys. Lett., 2012, 100, 113305.

11 Y. Wang, T. Sun, T. Paudel, Y. Zhang, Z. Ren and K. Kempa, Nano Lett., 2012, 12, 440-445.

12 M. A. Green and S. Pillai, Nature Photonics, 2012, 6, 130-132.

13 D. Y. Jiang, W. M. Yang, Y. J. Liu, H. L. Liu and J. H. Teng, J. Mater. Chem. C, 2015, 3, 3552-3558.

14 A. Lenert, D. M. Bierman, Y. Nam, W. R. Chan, I. Celanović, M. Soljačić and E. N. Wang, Nat. Nanotechnol., 2014, 9, 126130.

15 X. Liu, T. Starr, A. F. Starr and W. J. Padilla, Phys. Rev. Lett., 2010, 104, 207403.

16 C. M. Watts, D. Shrekenhamer, J. Montoya, G. Lipworth, J. Hunt, T. Sleasman, S. Krishna, D. R. Smith and W. J. Padilla, Nature Photonics, 2014, 8, 605-609.

17 N. I. Landy, S. Sajuyigbe, J. J. Mock, D. R. Smith and W. J. Padilla, Phys. Rev. Lett., 2008, 100, 207402.
18 F. Costa, A. Monorchio and G. Manara, IEEE Trans. Antennas Propag., 2010, 58, 1551-1558.

19 Y. Liu, S. Gu, C. Luo and X. Zhao, Appl. Phys. A, 2012, 108, 1924.

$20 \mathrm{~W}$. Zhu, Y. Huang, I. D. Rukhlenko, G. Wen and M. Premaratne, Opt. Express, 2012, 20, 6616-6621.

21 F. Costa and A. Monorchio, IEEE Trans. Antennas Propag., 2012, 60, 2740-2747.

22 X. Chen, Y. Li, Y. Fu and N. Yuan, Opt. Express, 2012, 20, 28347-28352.

23 F. Ding, Y. Cui, X. Ge, Y. Jin and S. He, Appl. Phys. Lett., 2012, 100, 103506.

24 C. Long, S. Yin, W. Wang, W. Li, J. Zhu and J. Guan, Sci. Rep., 2016, 6, 21431.

25 R. Huang and Z. Li, Appl. Phys. Lett., 2012, 101, 154101.

26 W. Li, T. Wu, W. Wang, P. Zhai and J. Guan, J. Appl. Phys., 2014, 116, 044110.

27 Y. J. Yoo, S. Ju, S. Y. Park, Y. J. Kim, J. Bong, T. Lim, K. W. Kim, J. Y. Rhee and Y. P. Lee, Sci. Rep., 2015, 5, 14018.

28 P. Bonhôte, A.-P. Dias, N. Papageorgiou, K. Kalyanasundaramand and M. Grätzel, Inorg. Chem., 1996, 35, 1168-1178.

29 J. G. Huddleston, A. E. Visser, W. M. Reichert, H. D. Willauer, G. A. Broker and R. D. Rogers, Green Chem., 2001, 3, 156-164. 30 V. M. Petrov and V. V. Gagulin, Inorg. Mater., 2001, 37, 93-98. 31 J. P. Southall, H. V. S. A. Hubbard, S. F. Johnston, V. Rogers, G. R. Davies, J. E. McIntyre and I. M. Ward, Solid State Ionics, 1996, 85, 51-60.

32 A. B. McEwen, H. L. Ngo, K. LeCompte and J. L. Goldman, J. Electrochem. Soc., 1999, 146, 1687-1695.

33 V. Panwar and R. M. Mehra, Polym. Eng. Sci., 2008, 48, 21782187.

34 Y. Pang, J. Wang, Q. Cheng, S. Xia, X. Y. Zhou, Z. Xu, T. J. Cui and S. Qu, Appl. Phys. Lett., 2017, 110, 104103.

35 Y. Naito and K. Suetake, IEEE Trans. Microwave Theory Tech., 1971, 19, 65-72.

36 F. Yu, J. Wang, J. Wang, H. Ma, H. Du, Z. Xu and S. Qu, Appl. Phys. Lett., 2015, 107, 211906.

37 X. Chen, T. M. Grzegorczyk, B.-I. Wu, J. Pacheco Jr and J. A. Kong, Phys. Rev. E: Stat., Nonlinear, Soft Matter Phys., 2004, 70, 016608.

38 Q. Zhao, J. Zhou, F. Zhang and D. Lippens, Mater. Today, 2009, 12, 60-69. 